\title{
Contribuições da enfermagem na potencialização do processo de adaptação ao paciente com doença renal crônica
}

RESUMO | Objetivo: Identificar as ações assistenciais-educativas da enfermagem ao paciente com Doença Renal Crônica em uso de hemodiálise e como estas tem contribuído no processo de adaptação do paciente. Método: Trata-se de revisão integrativa da literatura realizada nas bases de dados da Literatura Latino-Americana (LILACS) e National Library of Medicine National Institutes of Health dos EUA (PUBMED), sendo selecionada a amostra de 11 artigos. Resultados: Os artigos encontrados descrevem as contribuições das ações assistenciais-educativas de enfermagem aos pacientes com Doença Renal Crônica em necessidade de hemodiálise, no que tange ao seu processo de adaptação e integridade. Conclusão: As condutas de enfermagem com maior grau de mudança e condução de melhores resultados foram as açães educativas, com consequentes redução de risco, redução da progressão de lesão renal.

Palavras-chaves: Cuidados de Enfermagem; Insuficiência Renal Crônica; Processo de Enfermagem.

ABSTRACT | Objective: To identify nursing care-educational actions to the patient with chronic renal disease using hemodialysis and how this has contributed to the patient's adaptation process. Methods: It is an integrative review of the literature carried out in the Latin American Literature (LILACS) and National Library of Medicine (PUBMED) databases, with a sample of 11 articles selected. Results: The articles found describe the contributions of care-educational nursing actions to patients with Chronic Renal Disease in need of hemodialysis, regarding their process of adaptation and integrity. Conclusion: The nursing conducts with greater degree of change and conduction of better results were educational actions, with consequent reduction of risk, reduction of the progression of renal injury. Keywords: Nursing Care; Renal Insufficiency; Chronic; Nursing Process.

RESUMEN | Objetivo: Identificar acciones de atención de enfermería y educación para el paciente con enfermedad renal crónica mediante hemodiálisis y cómo esto ha contribuido al proceso de adaptación del paciente. Metodos: Es una revisión integradora de la literatura realizada en las bases de datos de Literatura Latinoamericana (LILACS) y de la Biblioteca Nacional de Medicina (PUBMED), con una muestra de 11 artículos seleccionados. Resultados: Los artículos encontrados describen las contribuciones de las acciones de enfermería de cuidado y educación a pacientes con enfermedad renal crónica que necesitan hemodiálisis, en relación con su proceso de adaptación e integridad. Conclusión: Las conductas de enfermería con mayor grado de cambio y la conducción de mejores resultados fueron acciones educativas, con la consiguiente reducción del riesgo, reducción de la progresión de la lesión renal.

Palavras claves: Atención de Enfermería; Insuficiencia Renal Crónica; Proceso de Enfermería.

\section{Jaqueline Dantas Neres Martins}

Graduanda da Universidade do Estado do Pará.

\section{Dayara de Nazaré Rosa de Carvalho}

Enfermeira. Mestranda em Enfermagem da Universidade do Estado do Pará (UEPA).

\section{Daniele Melo Sardinha}

Enfermeira. Pós-graduanda em Cardiologia e hemodinâmica pela Faculdade Integrada da Amazônia (FINAMA).

\section{Annália de Paula Gesta Santos \\ Enfermeira.}

\section{Marcelo Williams Oliveira de Souza}

Enfermeiro. Doutorando do Programa de pós-graduação em Biologia de Agentes Infecciosos e Parasitários pela Universidade do Estado do Pará (UFPA).

\section{Viviane Ferraz Ferreira de Aguiar}

Enfermeira. Doutoranda no Programa de Pós-Graduação em Doenças Tropicais, Núcleo de Medicina Tropical/Universidade Federal do Pará. Docente da Faculdade de Enfermagem/Universidade Federal do Pará. INTRODUÇÃO:

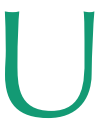
$\mathrm{m}$ dos grandes desafios do Sistema de Saúde nas últimas décadas é o despontamento da incidência de Doença Renal Crônica (DRC) no Brasil. Segundo a Sociedade Brasileira de Nefrologia há aumento de 40.000 pacientes anualmente com a doença ${ }^{1-6}$.

Ser portador de DRC e dependente de hemodiálise incorre em conviver diariamente com um arcabouço de riscos e probabilidade de desfechos clínicos que frequentemente serão estímulos ao paciente para vivenciar respostas adaptativas ou inefetivas, requerendo a utilização
Recebido em: 15/07/2019

Aprovado em: 28/08/2019

de mecanismos de enfrentamento ${ }^{6-9}$.

Neste contexto, torna-se fundamental a teoria de Callista Roy no processo de adaptação, visando melhor qualidade de vida do paciente. Para Roy considera-se adaptação a manutenção da integridade ou totalidade obtida por meio de metas que propiciem o seguimento dos ciclos biológicos intrínsecos a sobrevivência. Consequentemente, os mecanismos de enfrentamento são o elo fundamental para garantir respostas adaptativas do paciente, pois garantirão a integridade fisiológica e psíquica do indivíduo ${ }^{7-9}$.

Devido ao contexto de mudanças adaptativas a qual o paciente com doen- 
ça renal crônica é envolvido decorrente das alterações fisiológicas e interferências desses nas esferas psíco-emocional-social é importante a atuação da enfermagem na otimização das respostas do paciente frente a esses processos ${ }^{8-10}$.

As implicações da doença e da hemodiálise aos pacientes com doença renal crônica justificam a importância deste estudo, especialmente por este processo estar facilmente interligado na exteriorização de respostas inefetivas que podem culminar em graves desfechos clínicos. Tais condições possuem relevância na assistência de enfermagem a fim de favorecer respostas eficientes no paciente $\mathrm{e}^{11-13}$. Sendo assim, traz como questão de pesquisa: Quais as ações assistenciais educativas realizadas por enfermeiros a pacientes com Doença Renal Crônica em hemodiálise e como estas têm favorecido o processo de adaptação do paciente? Para tanto, delimitou-se como objetivo do presente estudo identificar as ações assistenciais-educativas da enfermagem e suas contribuições no processo de adaptação ao paciente com DRC em uso de hemodiálise.

\section{MÉTODO}

Estudo descritivo, do tipo Revisão Integrativa de Literatura (RIL). A RIL foi desenvolvida em seis etapas: formulação de objetivo e pergunta de pesquisa; estipulação de critérios para a obtenção de amostra; inclusão de artigos pertinentes à temática; análise dos artigos; discussão e resultados sintetizados e apresentação da revisão14. A busca nos bancos de dados e coleta ocorreu no primeiro semestre de 2019.

A busca dos artigos ocorreu em bases de dados da Literatura Latino-Americana (LILACS) e National Library of Medicine National Institutes of Health dos EUA (PUBMED). Utilizou-se Descritores em Ciências da Saúde (DECS), palavras chaves em inglês e português, pois as duas opções de idioma permitiram ampliação de estudos. As palavras chaves empregadas foram: doença renal crônica; adaptação; cuidados de enfermagem e DECS: nursing care; renal insufficiency, chronic.
Para efetivar a busca anexou-se tais termos ao conector boleano AND.

Para fins de operacionalização, foram selecionados os seguintes critérios de inclusão: artigos originais completos; disponibilidade na íntegra; publicados nos anos 2009 - 2018; idiomas em inglês, português e espanhol, que abordassem sobre assistência de enfermagem ao paciente com doença renal crônica em hemodiálise no título e/ ou resumo e ações assistenciais-educativas da enfermagem.

Com a utilização dos critérios de busca encontrou-se 367 artigos. Mediante a leitura dos resumos apenas 16 artigos encaixaram-se nos objetivos. Os demais artigos foram excluídos pelos motivos: indisponíveis; não abordavam sobre assistência de enfermagem ao paciente com DRC. Após a leitura na íntegra, houve a exclusão de mais 5 artigos: 2 artigos retratavam somente sobre o desenvolvimento de tecnologias ao paciente com DRC, 2 artigos tinham o foco no desenvolvimento de complicações e sintomatologias do paciente, no entanto, não abordavam assistência ao paciente e outro apresentava como abordagem a diálise peritoneal.

A priori foram incluídos na pesquisa os artigos que descreviam ações de enfermagem educacional e assistencial ao paciente com DRC em hemodiálise e que traziam colaborações positivas das ações na meIhora clínica e psicoemocional do cliente.

Dessa forma, para o presente estudo obteve-se o total de 11 artigos. A coleta de dados ocorreu por meio do formulário de URSI, a qual foi adaptado para a presente pesquisa com a seleção das variáveis contidas nos artigos: Título, ano, nome do periódico, base de dados, objetivos do estudo e principais resultados.

\section{RESULTADOS}

Esta pesquisa embasou-se na seleção de 11 artigos que descrevem a assistência de enfermagem a pacientes com DRC em hemodiálise. No quadro 1 são apresentados os artigos destacados. Desses, 2 artigos (18\%) eram de pesquisas interna- cionais, a saber: Nova Zelândia e Nova Escócia. Os demais, 82\% provinham do Brasil, sendo que respectivamente 4 (45\%), 3 (33\%), 2 (22\%) provinham das regiões nordeste, sudeste e sul.

A região norte não apresentou estudos relacionados à temática, fato atribuído às hipóteses: Menor aporte para densidade tecnológica e menor incentivo a estudos correlacionados, atribuído ao fato de ser região com menor concentração de pacientes com DRC em comparação as demais, de acordo com informações obtidas pelo Censo de diálise da Sociedade Brasileira de Nefrologia ${ }^{15}$.

Os artigos internacionais foram permeados de inovação e trouxeram grandes contribuições, em especial, no aspecto referente ao desenvolvimento de intervenções e tecnologias concernentes a autogestão e a integração do paciente neste processo.

Em relação ao eixo assistencial-educativo, 63\% (6) das pesquisas centralizavam na oferta de serviços intrínsecos a feição assistencial e 37\% (4) das demais pesquisas eram de constituição educativa, com finalidades de promover mudanças de comportamento e aperfeiçoar o processo de adaptação. A seguir encontra-se a figura $1 \mathrm{com}$ a síntese dos artigos encontrados.

\section{DISCUSSÃO}

Os artigos encontrados contribuíram ao esclarecer as principais dificuldades do paciente com DRC em necessidade de hemodiálise, no que tange ao seu processo de adaptação e esforço para manter a integridade ${ }^{12,16-17-20}$.

No Brasil, parte dos pacientes adentram ao sistema de saúde quando a severidade da patologia exige o procedimento de hemodiálise em caráter de urgência. Entretanto, o grau de estímulos na dependência de hemodiálise é superior ao de um paciente diagnosticado nas fases iniciais da patologia. Pois, o risco do procedimento hemodialítico, incorre em instabilidades hemodinâmicas, riscos de infecção, complicações da fístula arteriovenosa ${ }^{18-24}$.

Dessa maneira, as dificuldades en- 
Figura 1: Síntese dos artigos selecionados para o estudo, mediante título, ano, periódico, base de dados, objetivos, principais resultados. Belém, 2019.

\section{TíTULO \\ ANO/ PERIÓDI- \\ CO/ BASE DE DADOS}

1. Intervenção musical sobre ansiedade e parâmetros vitais de pacientes renais crônicos: ensaio clínico randomizado

2. Crianças e adolescentes renais crônicos em espaço educativoterapêutico: Subsídios para o cuidado cultural de enfermagem.

3. Intervenção educacional de enfermagem para redução da hiperfosfatemia em pacientes em hemodiálise.

\section{Using the Frailty Assessment} for Care Planning Tool (FACT) to screen elderly chronic kidney disease patients for frailty: the nurse experience.
2018, Revista

latino-americana

de enfermagem,

PUBMED.

\section{2, Revista da}

Escola de enfer-

magem da USP,

PUBMED.

2017, Revista

brasileira de enfer-

magem, PUBMED.
2018, Clinical Interventions in Aging, PUBMED
OBJETIVOS

Avaliar o efeito da intervenção musical na ansiedade e parâmetros vitais.

Descrever o cuidado educativo terapêutico no enfoque da enfermagem transcultural

Avaliar a efetividade de ação educacional de enfermagem para redução da hiperfosfatemia em pacientes renais crônicos em hemodiálise

\section{PRINCIPAIS RESULTADOS}

Os pacientes do grupo controle com efeito da musicoterapia antes da diálise tiveram redução da ansiedade, frequência cardiorrespiratória.

A intervenção terapêutica educacional da enfermeira trouxe maior conforto na diálise, aprendizado, valorização das subjetividades e particularidades.

Houve redução de fósforo e creatinina no início e após 30 dias após a realização da intervenção, assim como redução do prurido.
Explorar a experiência de enfermeiros de triagem para a fragilidade utilizando a ferramenta FACT em uma clínica
A utilização da ferramenta possibilitou avaliar o prognóstico, descrever as fragilidades e direcionar ações específicas equitativas as vulnerabilidades do paciente.
5. A prospective clinical trial of specialist renal nursing in the primary care setting to prevent progression of chronic kidney: a quality improvement report

6. Necessidades de orientação de enfermagem para 0 autocuidado de clientes em terapia de hemodiálise.
2014, BMC family practice, PUBMED
Descrever intervenção de melhoria da qualidade para um grupo de pacientes com DRC.
As intervenções de enfermagem possibilitaram redução do risco cardiovascular, colesterol e pressão arterial.
2010, Revista Brasileira de Enfermagem, LILACS.
Identificar as necessidades de orientação de enfermagem para o autocuidado de clientes em hemodiálise
Destacou-se um quantitativo significativo de pacientes em sistema de cuidado totalmente compensatório.
7. Pacientes renais crônicos em hemodiálise: um estudo sobre o modo psicossocial da teoria de Roy.
2014, Revista de pesquisa cuidado é fundamental, LILACS
Identificar os problemas adaptativos do modo psicossocial do modelo de Roy em pacientes renais crônicos em hemodiálise.
Houve predomínio de problemas relacionados a autoestima, função sexual nos pacientes com doença renal crônica.
8. Nursing diagnoses and adaptation problems among chronic renal patients.
2015, Investigación y Educación en Enfermería, LILACS.
Identificar semelhanças entre a NANDA International diagnósticos de enfermagem e modelo de adaptação de Roy entre pacientes renais submetidos a hemodiálise
Houve similaridade entre os conceitos e problemas enquadrados nos eixos de adaptação propostos por Callista Roy.
9. As intervenções de enfermagem mais prevalentes em um serviço de hemodiálise frente as intercorrências com a fístula arteriovenosa.
Descrever as intercorrências com a

2011, Nursing, fístula arteriovenosa durante sessão de LILACS. hemodiálise, identificar as intervenções de enfermagem mais prevalentes.
As intervenções de enfermagem mais utilizadas foram direcionados as complicações da fistula, alivio da dor e conforto do paciente.
10. Avaliação da intensidade da dor de pacientes renais crônicos em tratamento hemodialítico.
2016, Revista dor, Analisar a dor de pacientes renais LILACS. crônicos em tratamento hemodialítico.

Identificou-se que metade dos pacientes utilizam fármacos para alívio da dor, sem prescrição. 
TítULO

11. Ensaio clínico para o controle da ingesta hídrica de pacientes em tratamento hemodialítico.

\section{ANO/ PERIÓDICO/} BASE DE DADOS

2018, Revista latino-americana de enfermagem, PUBMED.
OBJETIVOS

Analisar o impacto de uma intervenção em pacientes em uso de hemodiálise no controle de líquidos.
PRINCIPAIS RESULTADOS

Pacientes do grupo controle conseguiram atingir as metas na redução do peso no período interdialítico.

Fonte: Dados da pesquisa.

contradas na otimização da resposta do paciente correspondem: a falta de engajamento do paciente no seu processo saúde-doença, déficit de conhecimento do paciente sobre a complexidade da patologia e suporte social inadequado, os quais repercutem na não adesão do tratamento e mudança de comportamento. Condições socioeconômicas, fatores culturais, baixa escolaridade influenciam o entendimento do paciente acerca da condição de saúde e são impeditivos da adesão, efetividade de acesso aos serviços de saúde 19,21-23. $^{\text {. }}$

Os artigos encontrados descrevem que os pacientes dependentes de hemodiálise portam mais estímulos e obstáculos no enfrentamento da doença, decorrentes de maior sobrecarga de estresse, portanto frequentemente manifestam as respostas: fadiga, perfusão tissular inadequada, hipóxia tecidual, privação de sono, ansiedade, náusea, vômitos, proteção ineficaz, prurido, infecção, desequilíbrio acidobásico, dessaturação de oxigênio, câimbra, hipotensão/ hipertensão, dor, desconforto, ganho de peso interdialítico, impotência, baixa autoestima, enfrentamento ineficaz. Essas respostas são comumente encontradas nesse grupo de pacientes, algumas delas são dignas de intervenção imediata e outros necessitam de acompanhamento contínuo para averiguar a eficiência da resposta e dos mecanismos de enfrentamento ${ }^{18-24}$.

Frente a essas prerrogativas, o cuidado é um dos elementos essenciais para aperfeiçoar as respostas do indivíduo, na qual a função deste processo incube ao enfermeiro. Através de suas competências e da ferramenta processo de enfermagem, o enfermeiro julgará as respostas do indivíduo a luz científica e clínica e produzirá um plano de cuidado correspondente ${ }^{1-6}$.
A assistência de enfermagem pautada no processo de adaptação implica em identificar vulnerabilidades, deficiências, déficits no autocuidado no indivíduo, promover intervenções no contexto fisiológico e nas dimensões biopsicossocial e familiar ${ }^{17-21}$.

De maneira geral, as ações assistenciais-educativas de enfermagem identificadas nos artigos foram: apoio sócio psicoemocional, acolhimento e escuta qualificada, supervisão do tratamento, o acompanhamento do regime terapêutico, promoção de estímulo das capacidades e incentivo das potencialidades do paciente, comunicação e negociação com o cliente sobre mudanças de comportamentos e atitudes, orientações sobre a importância do autocuidado e o seguimento da terapêutica, monitoração das respostas fisiológicas, controle dos parâmetros fisiológicos na hemodiálise, cooperação com o paciente e inclusão dos entes queridos do paciente nesse processo ${ }^{18-24}$.

Os principais cuidados prestados ao paciente na hemodiálise foram o controle dos sinais vitais, acompanhamento das reações e o ajuste do paciente em casos de instabilidades hemodinâmicas, adjunto do emprego de terapias complementares, ambos colaboraram na indução de respostas adaptativas e suporte ao mecanismo de enfrentamento, como o controle dos parâmetros vitais: Frequência cardíaca, frequência respiratória e redução da ansiedade, ou seja, minimização das possibilidades de descompensações hemodinâmicas, culminando na integridade estrutural-psíquica e otimização dos modos adaptativos correspondente ao modo fisiológico e função de papel ${ }^{5,6,19-25}$.

As ações educativas destacadas nos estudos foram subsidiadas por intermédio da comunicação ativa com o paciente e orientação pautada na construção de tecnologias educacionais as quais permitiram enquadrar metas e comportamentos indispensáveis para o alcance dos resultados esperados: respostas adaptativas ${ }^{20-25}$.

As ações cuidativas-educativas de enfermagem constituídas por meio de tecnologias facilitaram a compreensão dos pacientes sobre a mudança de comportamento, redução do consumo de sal, controle da dieta, redução do consumo de líquidos, que a priori contribuiu para melhor adesão ao tratamento e produção de respostas: redução do peso no período interdialítico, correção da hiperfosfatemia, estabilização da pressão arterial, conforto, conforto, equilíbrio de fluídos e eletrólitos, funções de eliminação, oxigenação, padrão cardíaco estabilizado e redução de fragilidades. Concomitamente pode-se afirmar que tais favoreceram respostas adaptativas, pois proporcionaram correção dos parâmetros antropométricos, hidroeletrolítico e controle da sobrecarga de estresse no organismo ${ }^{23-24}$. Além disso, por intermédio dessas ferramentas educacionais possibilitou cuidado integral pautado nas necessidades humanas com a inserção das vulnerabilidades.

Em conformidade, outros estudos também elucidam a relevância e o impacto das ações educativas para aprimoramento das capacidades humanas, melhor adesão ao tratamento, melhora da sobrevida e redução dos riscos intrínsecos a patologia e as contribuições da enfermagem na redução de riscos cardiovasculares e eventos desfavoráveis ${ }^{20-25}$.

Os estudos enfatizados, dos países da Nova Zelândia ${ }^{16}$ e Nova Escócia ${ }^{17}$ descrevem também a interconexão de enfermeiros das diferentes redes de atenção 
à saúde, como aspectos imprescindíveis que propuseram a captar precocemente o grupo de pacientes com DRC para intervenção, garantindo melhor sobrevida, além de que possibilitou a inclusão de ferramentas as quais permitem avaliar as fragilidades do paciente elaborar e alternativas vigentes à situação.

\section{CONCLUSÃO}

A revisão integrativa da literatura permitiu identificar que as ações assistenciais-educativas de enfermagem ao paciente com DRC são inexoráveis para impulsionar o processo de adaptação do paciente. No que refere ao presente estudo, as ações assistenciais-educativas de enfermagem foram principalmente: supervisão e monitorização do paciente no processo hemodialítico, intervenções educativas de estímulo as capacidades e potencialização das habilidades e fortalezas do cliente, assim como a negociação e comunicação fluida a fim de solidificar relacionamento interpessoal para a criação de vínculos e possibilitar maior apurácia na coleta de dados e estabelecimento de plano de cuidados eficaz.

Dessa forma, as ações assistenciais oportunizaram o acompanhamento das reações dos pacientes no processo hemodialítico e intervenção imediata em casos de desajustes, por fim corrigiu a iminência de estímulos/respostas nocivos ao indivíduo. As ações educativas, preencheram lacunas de conhecimento, capacitaram e viabilizaram o processo de cuidar, sustentação da mudança de comportamentos e fortalezas no enfrentamento de fragilidades.

\section{Referências}

1. Ferreira LA, Trindade TA, Chouchonova NSN, Augusto FM, Lourdes IR, ValadaresWOJ, et al. Prevalence of chronic kidney disease in a city of southeast Brazil. J Bras Nefrol. 2017; 39 (2): 126-134. DOI: 10.5935/0101-2800.20170030.

2. Novakowski DS, Ferraz ET, Antonia MRC, Maran E, Rosa RS, Moreira NA. Accessibility to treatment and health status of hemodialytic patients. Rev Enferm UFPE on line. 2018; 12 (7): 1853-8. DOl: https://doi.org/10.5205/1981-8963-v12i7a23468 5p1853-1858-2018.

3. Herrera PA, Benites VZ, Hernandez AV, Mezones EH, Silveira MC. Mortality in patients with chronic kidney disease undergoing hemodialysis in a public hospital of Peru. J Bras Nefrol. 2018; 35 (2): 192-7. DOl: http://dx.doi.org/10.5935/0101-2800.20150031. 4. Maria CFQ, Isabel MCDF, Graças MMN, Dantas JS, Venícios MOL, Luisa ABCL. Components of a roy's adaptation model In patients undergoing hemodialysis. Rev. Gaúcha Enferm. 2013; 34 (4): 45-52. DOI: http://dx.doi.org/10.1590/5198314472013000400006

5. Duarte NBG, Pessoa NRL, Jeane CLP, Pereira KM, Rodrigues GSF, Neyla KFMC. Quality of life of men and women on hemodialysis. Rev Baiana Enferm. 2018; 32. DOI: 10.18471/rbe.v32.24935.

6. Andrade IR, Valéria FCS, Silva TC, Bertolossi CM, Abrantes RL. The Caring Costs for Patients Bearing Chronic Kidney Disease (CKD), in a Non-Dialytic Phase of a University Hospital. J. Res.: Fundam. Care. Online. 2018; 10 (3): 647-655. DOI: 10.9789/21755361.2018.v10i3.647-655.

7. Filipe AAJC. Fluid and dietary restriction's efficacy on chronic kidney disease patients in hemodialysis. Rev Bras Enferm. 2015; 68 (6). DOl: http://dx.doi.org/10.1590/ 0034-7167.2015680622i.

8. Maria CFQF, Graças MMNP, Dantas JS, Maria CBB, Beatriz FBLS, Luisa ABCL. Chronic kidney patients in hemodialysis: a study on the mode of psychosocial theory of Roy. J. Res.: Fundam. Care. Online. 2014; 6 (4): 1455- 1463. DOI: 10.9789/2175-5361.2014. v6i4.1455-1463.

9. Maria SSBL, Luzia JL, Godoy S, Martins JABT, Gonçalves RR, Valéria FCF. Treatment adherence of chronic kidney disease patients on hemodialysis. Acta Paul Enferm. 2018; 31 (1):54-60. DOl: http://dx.doi.org/10.1590/1982-0194201800009.

10. Peixoto VSP, Veraci MOQ, Salete MBJ. Children and adolescents with chronic kidney disease in an educational therapeutic environment: support for cultural nursing care. Rev Esc Enferm USP. 2012; 46 (5): 1057-1065. DOI: http://dx.doi.org/10.1590/S008062342012000500004

11. Maria CFQF, Beatriz AAM, Graças MMNP, Cruz BE, Venícios MOL, Luisa ABCL. Nursing diagnoses and adaptation problems among chronic renal patients. Invest Educ Enferm. 2015; 33 (1). Disponível em: < http://www.scielo.org.co/scielo.php?script=sci_arttext\&pid=S0120-53072015000100014>.

12. Miladi EFS, Maria RK, Azevedo LG, Raquel ERB, Aparecida DB. Educational nursing intervention to reduce the hyperphosphatemia in patients on hemodialysis Intervención educativa de enfermería para reducir hiperfosfatemia en pacientes en hemodiálisis. Rev Bras Enferm. 2017; 70 (1):31-8. DOl: http://dx.doi.org/10.1590/0034-7167-2016-0015.
13. Alcântara GAM, Bezerra AR, Alves MF, Sandro AMG, Peres PO, Áfio JC. Intervenção musical sobre a ansiedade e parâmetros vitais de pacientes renais crônicos: ensaio clínico randomizado. Rev. Latino-Am. Enfermagem. 2018; 26. DOI: 10.1590/15188345.2123.2978.

14. Dal KSM, Cristina RCPV, Maria CG. Integrative literature: a research method to incorporate evidence in health care and nursing. Texto Contexto-Enferm. 2008; 17 (4): 758-64. DOl: http://dx.doi.org/10.1590/S0104-07072008000400018.

15. Sociedade Brasileira de Nefrologia. Censo de 2017. Censo de diálise revela $40 \mathrm{mil}$ novos pacientes em 2017 no País. 2018. Disponível em <https://arquivos.sbn.org.br/ uploads/sbninforma114.pdfs.

16. Walker RC, Marshall MR, Polaschek NR. A prospective clinical trial of specialist renal nursing in the primary care setting to prevent progression of chronic kidney: a quality improvement report. BMC Family Practice. 2014; 15. DOI: https://doi. org/10.1186/1471-2296-15-155.

17. Moffat $H$, Moorhouse P, Mallery L, Landry D, Tennankore K. Using the Frailty Assessment for Care Planning Tool (FACT) to screen elderly chronic kidney disease patients for frailty: the nurse experience. Clin. Interv. Aging. 2018; 13: 843-852. DOl: https://doi. org/10.2147/CIA.S150673.

18. Santos I, Paula RFR, Márcia LMB. Needs of nursing guidance for self-care of clients on hemodialysis therapy. Rev Bras Enferm. 2011; 64 (2): 335-342. DOl: http://dx.doi. org/10.1590/S0034-71672011000200018.

19. Allana GSAOO, Pilotto MO, Bernardi CC, Regina CST, Abrão JCC, Kusumota L. Ensaio clínico para o controle da ingestão hídrica de pacientes em tratamento hemodialítico. Rev. Latino-Am. Enfermagem. 2018; 26. DOl: http://dx.doi.org/10.1590/15188345.2694.3091.

20. Silva CJ, Beuter M, Marilene NOGP, Schwartz E, Tambará ML, Castro CR. Family dynamics of elderly members undergoing pré- dialysis treatmente. Esc Anna Nery. 2017 21 (1). DOl: http://dx.doi.org/10.5935/1414-8145.20170023.

21. Lidyane FLN, Rodrigues LF, Silva NC, Peixoto VSP. Percepção do paciente renal crônico acerca dos cuidados com acessos para a hemodiálise. Cogitare Enfermagem. 2016; 21 (3): 01-8 DOI: http://dx.doi.org/10.5380/ce.v21i3.45628.

22. Thaís LMS,Gomes MB. Developing educational material on chronic kidney disease using best practices in health literacy. J Bras Nefrol. 2017; 39 (1): 55-8. DOI: 10.5935/0101-2800.20170009.

23. Peeters MJ, Van ADZ, Van JDM, Bots ML, Van MB, A MTD et al. Nurse Practitioner Care Improves Renal Outcome in Patients with CKD. J Am Soc Nephrol. 2014; 25 (2): 390-8. DOI: 10.1681/ASN.2012121222.

24. Rita AB, Cavalheiro CMG, Fátima MMC,Souza C, Henrique DT,Costa KF et al. Hemodialysis services: are public policies turned to guaranteeing the access?. Cad. Saúde Pública. 2015; 13 (7): 1505-1516. DOl: http://dx.doi.org/10.1590/0102-311X00073514. 25. Wang J, Yue $P$, Huang J, Xie $X$, Ling $Y$, Jia LYX, et al. Nursing Intervention on the Compliance of Hemodialysis Patients with End-Stage Renal Disease: A Meta-Analysis. Blood Purif. 2018; 45: 102-9. DOl: 10.1159/000484924. 